\title{
Survey of Medical Student Empathy at a Canadian Medical School: A Cross-Sectional Quantitative Survey
}

Safina Adatia, MD, MSc | Pierre-Paul Tellier, MD, CFPC | Raheem Remtulla, MD, BHSc PRiMER. 2022;6:6.

Published: 3/8/2022 | DOI: 10.22454/PRiMER.2022.535629

\section{Abstract}

Introduction: Within a medical context, empathy is defined as "an appropriate understanding and communication of a patient's experience." While it has been established that empathy is an important quality to have as a doctor, studies have shown that empathy in medical students declines during their clinical years. However, there are no studies to date that evaluate medical student empathy in Canada. Therefore, we aimed to evaluate medical student empathy at McGill University Medical School using the Jefferson Scale of Empathy (JSE).

Methods: We used a cross-sectional study design and invited medical students across all 4 years, in October 2019, to complete the JSE. The JSE is a validated psychometric tool that measures empathy at one point in time. The survey was distributed via email and on social media.

Results: A total of 133 students from all 4 years responded, proportionate across each year; 119 responses were included in analysis. Differences in mean questionnaire were not statistically significant for gender, age or specialty interest. The analysis of variance for differences in year of medical school was significant $(P=.0104)$. Between groups analysis revealed a statistically significant decrease between Med-2 empathy scores (average score 117.6) and Med-3 (107.5), $P<.01$. Multivariable analysis demonstrated the decrease in empathy remained statistically significant $(P<.05)$.

Discussion: Our statistical analysis determined that medical students' empathy declines between the second and third year of medical school in a Canadian context, consistent with global results. This information can help target changes in the medical curriculum to preserve empathy in students, and prevent this decline, which could then be applied to other medical schools internationally.

\section{Introduction}

Empathy is an important quality in a physician. It has been outlined as such by the CanMEDS framework developed by the Royal College of Physicians and Surgeons of Canada, and the College of Family Physicians of Canada, the professional organizations that drive medical school and residency curricula in Canada. ${ }^{1}$ No single, unified definition of empathy currently exists; however, a commonly-cited definition within medical literature is "an appropriate understanding and communication of the patient's experiences." ${ }^{2}$ 


\section{Empathy in Medical Clinical Practice}

The CanMEDS framework states that physicians must establish a therapeutic relationship with their patients, and communicate using empathy and a patient-centered approach. ${ }^{1,3}$ Benefits to incorporating empathy into physician practice include improvements in patient satisfaction, trust, and comfort, ${ }^{4-7}$ along with decreases in patient anxiety and stress, better clinical outcomes, and self-efficacy and shared decision-making. ${ }^{8,9}$

Furthermore, doctors who are empathetic experience higher rates of job satisfaction and mental health, ${ }^{10}$ make better clinical decisions, ${ }^{11-14}$ and are more effective leaders in their field. ${ }^{15}$

However, despite the fact that it constitutes an important aspect of empathy, current evidence points to a decreased interest in good physician-patient communication in medical general practice. ${ }^{8}$

\section{Empathy in Undergraduate Medical Education}

It has been demonstrated that over the 4 years of medical training, attitudes of students toward patient suffering may change negatively. ${ }^{16,17}$ Using the Jefferson Scale of Empathy (JSE), it was found that empathy significantly declined during the third year (known as clerkship) of medical school. ${ }^{18}$ McGill University's medical school has implemented a core component of the curriculum, known as physicianship, that addresses concerns related to empathy, among other skills, though the goal of the development of the program was not to prevent drops in physician empathy. ${ }^{19}$ While such a program has existed for many years, there has not been an assessment of students' empathy over the course of the 4 years of medical training.

This study aims to fulfill this knowledge gap. In this investigation we aimed to assess empathy levels according to the Jefferson Scale of Empathy among McGill medical students and analyze characteristics such as age, gender, speciality of interest, and year in medical training that may be associated with differences among students.

\section{Methods}

\section{Research Design and Context}

This study used a cross-sectional survey. The survey was administered to students in late October of the school year, in order to allow time for transition between years. We allowed 4 weeks for the collection of data.

The population we targeted was composed by the totality of medical students at McGill University enrolled in the academic year 2018-2019.

\section{Data Gathering}

The survey was distributed via email and on social media. We encouraged participation by offering prizes to participants. We used the online software platform SurveyMonkey to collect our data.

The instrument that we used to measure empathy in McGill medical students is the JSE. The JSE is a reliable, validated, international tool used to assess empathy in health professions students. ${ }^{20}$ While the scale has been used to assess student empathy internationally, to our knowledge it has never been used in the Canadian medical student context.

\section{Data Analysis}

We computed descriptive statistics and their intervals per cohort. We used an analysis of variance (ANOVA) to compare mean empathy scores between groups for gender (male vs female vs other), age (less than 22 years to represent medical students accepted directly from preuniversity colleges vs more than 22 years for all other students), specialty interest (medical specialties vs surgical specialties vs undecided) and year in medical 
school (Med 1 vs Med 2 vs Med 3 vs Med 4).

We compared demographic variables in a univariable analysis ( $\chi^{2}$ test). If demographic variables were found to have a statistically significant relationship with empathy scores, we conducted a multiway ANOVA to determine if there was signifcance. We included variables with $P<.10$ in the univariable analysis with statistical significance in the multiway ANOVA. We used JMP to conduct the analysis.

We obtained ethics approval from the McGill Faculty of Medicine Research and Ethics Compliance Board.

\section{Results}

\section{Descriptive Statistics}

A total of 133 of 730 students responded; 16 responses were removed from the analysis due to incompletion. This resulted in a total of 119 included responses: 37 from Med 1 (21\% of the cohort), 30 from Med 2 (17\%), 22 from Med 3 (12\%), and 30 from Med 4 (17\%); 80 respondents were female, and 37 were male, with one preferring not to answer and one transgender male.

\section{Statistical Analysis}

Differences in questionnaire mean were not observed for gender $(P=.364)$, age $(P=.498)$ and specialty interest $(P=.436)$. The ANOVA for differences in year of medical school was significant $(P=.0104)$. Between-groups analysis revealed a statistically significant difference between Med 3 empathy scores (average score 107.5) and Med 2 (117.6), $P<.01$. In the univariable analysis of demographic factor, there was a statistically significant relationship between year of medical school with desired specialty and age $(P<.05)$. There was no relationship between year of medical school and gender. A three-way ANOVA comparing year of medical school, desired specialty, and age with empathy scores was still statistically significant $(P<.05)$, indicating no significant interaction between these factors and the effect of year on empathy score.

\section{Discussion}

This study evaluated medical student empathy scores at McGill University. The goal of the study was to assess differences in empathy based on gender, age, specialty of interest and year in medical school. While gender, age, and desired specialty did not result in significant differences, year in medical school did. Specifically, we found a decline in empathy score between Med 2 and Med 3.

These results mirror findings found in other medical student empathy studies, where students seem to experience a drop in empathy as they begin their clinical, in-hospital training. ${ }^{21,22}$ There are various potential reasons as to why this may happen. A systematic review conducted in Germany in 2011 by Neumann et al, ${ }^{17}$ investigated and summarized reasons for changes in medical student empathy. This review found that students who were more interested in "patient-oriented" specialties had higher rates of empathy compared to those who were interested in "patient-remote" specialties (surgery, radiology), ${ }^{17}$ though we did not find such a difference. The authors also found that "distress," which included burnout, lack of sleep, depression, low selfworth and reduced quality of life, influenced self-assessed empathy in students, and is in fact the main reason for such an empathy decline. These could potentially explain our finding of a decrease in empathy during clerkship. Qualitative analysis examining why students experience a decline in empathy within a Canadian context would be useful to better understand our findings. Longitudinal curriculum components like physicianship could provide the platform necessary to intervene and prevent this decline from happening, though at this time it does not seem to be protective. ${ }^{19}$

Interestingly, our study differed from other medical student empathy studies in that mean empathy scores 
increased after third year (mean empathy score in Med 3, 107.5 and in Med 4, 115.3). However this difference was not statistically significant. These findings may be attributed to the fact that most of Med 4 includes elective rotations and didactic teaching, hence less stressful. Moreover, the final year of the unique longitudinal physicianship curriculum may include components that reinforce the importance of empathy.

Limitations to our study include the fact that the empathy scores are self-reported and subjective. Additionally, our results represent only $16 \%$ of all medical students, though we did have similar response rates among the various years. Finally, this is a quantitative study and we are unable to draw conclusions as to why empathy declines. Qualitative research into the changes that occur between second and third year could explain these.

\section{Conclusion}

Empathy is an important quality necessary for a fruitful physician-patient relationship. This study helped us understand the change in empathy during medical school according to year, age, gender, and desired specialty after graduation at a Canadian medical school. Our study found a significant decline in empathy between the second and third years of medical school, consistent with results globally. It is currently unclear what factors into this decline. Further research with greater participation from the medical student body, and qualitative data will help us discover such information to potentially adapt the medical curriculum.

\section{Acknowledgments}

Funding: Author Safina Adatia received a research bursary of $\$ 2,000$ to compensate for time spent on the project. Author Pierre-Paul Tellier used residual funds (\$1,000, approximately) to fund this project.

Presentations: This study was presented as follows:

- International Association of Medical Education Conference (AMEE). Short communication on demand. August 2021.

- Family Medicine Forum (FMF). Poster presentation. November 2021

- Canadian Conference on Medical Education. Oral presentation. April 2022 (Accepted).

\section{Corresponding Author}

Safina Adatia, MD, MSc

Dept of Family Medicine, McGill University, 5858,Chemin de la Côte-des-Neiges, 3rd floor, Montreal, Quebec H3S 1Z1, Canada

safina.adatia@mail.mcgill.ca

\section{Author Affiliations}

Safina Adatia, MD, MSc - Department of Family Medicine, McGill University, Montreal, Quebec, Canada Pierre-Paul Tellier, MD, CFPC - Department of Family Medicine, McGill University, Montreal, Quebec, Canada Raheem Remtulla, MD, BHSc - Department of Ophthalmology, McGill University, Montreal, Quebec, Canada

\section{References}

1. CanMEDS: Better standards, better physicians, better care: Royal College of Physicians and Surgeons of Canada. Published 2018. Accessed February 22, 2022. http://www.royalcollege.ca/rcsite/canmeds /canmeds-framework-e

2. Eikeland H-L, Ørnes K, Finset A, Pedersen R. The physician's role and empathy - a qualitative study of third year medical students. BMC Med Educ. 2014;14(1):165. doi:10.1186/1472-6920-14-165 
3. Shaw EOI, Fowler N, eds. CanMEDS-FM 2017: A competency framework for family physicians across the continuum. The College of Family Physicians of Canada; 2017.

4. Thom DH; Stanford Trust Study Physicians. Physician behaviors that predict patient trust. J Fam Pract. 2001;50(4):323-328.

5. Krupat E, Bell RA, Kravitz RL, Thom D, Azari R. When physicians and patients think alike: patient-centered beliefs and their impact on satisfaction and trust. J Fam Pract. 2001;50(12):1057-1062.

6. Reis HT, Clark MS, Pereira Gray DJ, et al. Measuring responsiveness in the therapeutic relationship: a patient perspective. Basic Appl Soc Psych. 2008;30(4):339-348. doi:10.1080/01973530802502275

7. Kim SS, Kaplowitz S, Johnston MV. The effects of physician empathy on patient satisfaction and compliance. Eval Health Prof. 2004;27(3):237-251. doi:10.1177/0163278704267037

8. Derksen F, Bensing J, Lagro-Janssen A. Effectiveness of empathy in general practice: a systematic review. Br J Gen Pract. 2013;63(606):e76-e84. doi:10.3399/bjgp13X660814

9. Zachariae R, Pedersen CG, Jensen AB, Ehrnrooth E, Rossen PB, von der Maase H. Association of perceived physician communication style with patient satisfaction, distress, cancer-related self-efficacy, and perceived control over the disease. Br J Cancer. 2003;88(5):658-665. doi:10.1038/sj.bjc.6600798

10. Gleichgerrcht E, Decety J. The relationship between different facets of empathy, pain perception and compassion fatigue among physicians. Front Behav Neurosci. 2014;8:243.

doi:10.3389/fnbeh.2014.00243

11. Larson EB, Yao X. Clinical empathy as emotional labor in the patient-physician relationship. JAMA. 2005;293(9):1100-1106. doi:10.1001/jama.293.9.1100

12. Levinson W, Gorawara-Bhat R, Lamb J. A study of patient clues and physician responses in primary care and surgical settings. JAMA. 2000;284(8):1021-1027. doi:10.1001/jama.284.8.1021

13. Coulehan JL, Platt FW, Egener B, et al. "Let me see if I have this right...": words that help build empathy. Ann Intern Med. 2001;135(3):221-227. doi:10.7326/0003-4819-135-3-200108070-00022

14. Beckman HB, Frankel RM. Training practitioners to communicate effectively in cancer care: it is the relationship that counts. Patient Educ Couns. 2003;50(1):85-89. doi:10.1016/S0738-3991(03)00086-7

15. Skinner C, Spurgeon P. Valuing empathy and emotional intelligence in health leadership: a study of empathy, leadership behaviour and outcome effectiveness. Health Serv Manage Res. 2005;18(1):1-12. doi:10.1258/0951484053051924

16. Woloschuk W, Harasym PH, Temple W. Attitude change during medical school: a cohort study. Med Educ. 2004;38(5):522-534. doi:10.1046/j.1365-2929.2004.01820.x

17. Neumann M, Edelhäuser F, Tauschel D, et al. Empathy decline and its reasons: a systematic review of studies with medical students and residents. Acad Med. 2011;86(8):996-1009.

doi:10.1097/ACM.0b013e318221e615

18. Hojat M, Mangione S, Nasca TJ, et al. An empirical study of decline in empathy in medical school. Med Educ. 2004;38(9):934-941. doi:10.1111/j.1365-2929.2004.01911.x

19. Physicianship. McGill University. Accessed February 22, 2022. https://www.mcgill.ca/ugme/curriculum /curriculum-components/physicianship.

20. Kane GC, Gotto JL, Mangione S, West S, Hojat M. Jefferson Scale of Patient's Perceptions of Physician Empathy: preliminary psychometric data. Croat Med J. 2007;48(1):81-86.

21. Chen $D$, Lew R, Hershman W, Orlander J. A cross-sectional measurement of medical student empathy. J Gen Intern Med. 2007;22(10):1434-1438. doi:10.1007/s11606-007-0298-x

22. Chen DCR, Kirshenbaum DS, Yan J, Kirshenbaum E, Aseltine RH. Characterizing changes in student empathy throughout medical school. Med Teach. 2012;34(4):305-311.

doi:10.3109/0142159X.2012.644600 